\title{
In situ/operando Study of Photoelectrochemistry Using Optical Liquid Cell Microscopy
}

Khim Karki ${ }^{1}$, Pawan Kumar ${ }^{2}$, Antoine Verret ${ }^{2}$, Noah Glachman ${ }^{2}$, Daan Hein Alsem ${ }^{1}$, Deep Jariwala ${ }^{2}$, Norman Salmon ${ }^{1}$ and Eric Stach $^{2}$

${ }^{1}$ Hummingbird Scientific, Lacey, Washington, United States, ${ }^{2}$ University of Pennsylvania, Philadelphia, Pennsylvania, United States

Recent advances in liquid cell transmission electron microscopy (TEM) have enabled the study of reactions in liquid-solid interface at high spatial resolution in real-time environment. The capability to apply stimuli such as electrical, heating and electrochemical measurements has already started to provide new insights on the dynamics and structural changes during nanoparticles synthesis, lithium charge and discharge, crystal growth and metal corrosion. [1] The further ability to introduce light within the confined liquid environment of TEM strengthens the capability by allowing the study of light interaction in materials during reaction at solid-liquid interface, thus enabling in-situ photoelectrochemistry in the TEM. The study of hydrogen generation via photoelectrochemical method is one relevant application that can benefit from this technique and can provide fuel for energy storage solution in a clean and environmentally-friendly manner [2-4]. Particularly, the atomic scale mechanisms of the photocatalysts that facilitate the water splitting for efficient hydrogen generation are currently poorly understood. Further understanding of the role of various photocatalysts and the physics governing the active hydrogen evolution sites will allow for better and efficient design of photoelectrochemical devices.

We have developed a unique in situ/ operando liquid cell transmission electron microscope (TEM) holder with photo-capable light source [5] which can be used to study photocatalytic reactions in real time at nanometer length scales (Figure 1A and 1B). Using some of the model materials such as Au nanoprisms and $\mathrm{MoS}_{2}$ flakes, we will present the correlation of $\mathrm{I}-\mathrm{V}$ characteristics with water splitting and simultaneous structural changes at the catalytically active sites. These photocatalysts were chosen because the proposed mechanism for each exhibits a spatial dependence: plasmonically enhanced catalysis for Au nanoprisms and catalytically active edge sites for $\mathrm{MoS}_{2}$ flakes. For accurate quantitative information and reliability of materials interface in liquid, we will also present strategies for site-specific deposition/loading of sample materials in the desired electrodes of liquid cell chips for electroanalytical characterization and TEM imaging. An example of directly deposited $\mathrm{MoS}_{2}$ layers in the working electrode of cell is shown in Figure 1C. The redox IV characteristic of the sample with and without light (Figure 1D) shows enhanced peak currents in the case of light illumination, suggesting light-induced catalytic effect of the sample. The availability and utilization of photo stimuli in liquid cell TEM can provide important fundamental insights into the understanding of several other photoelectrochemical systems [5]. 
A

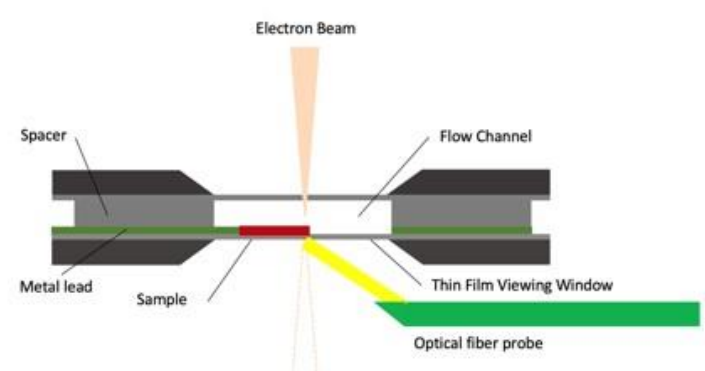

Transmitted Electrons

C

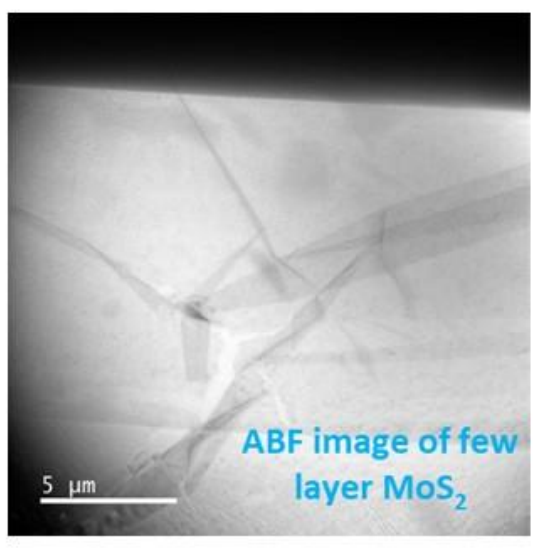

B

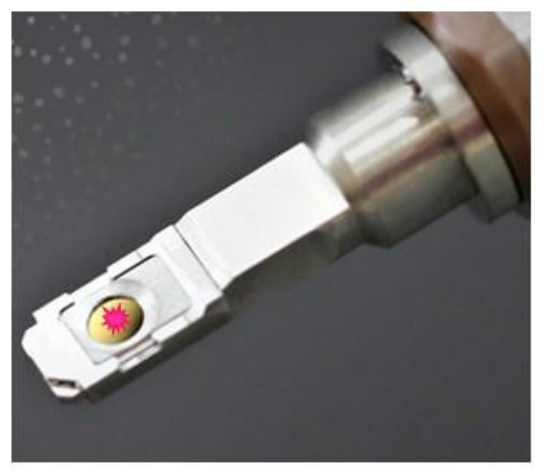

D

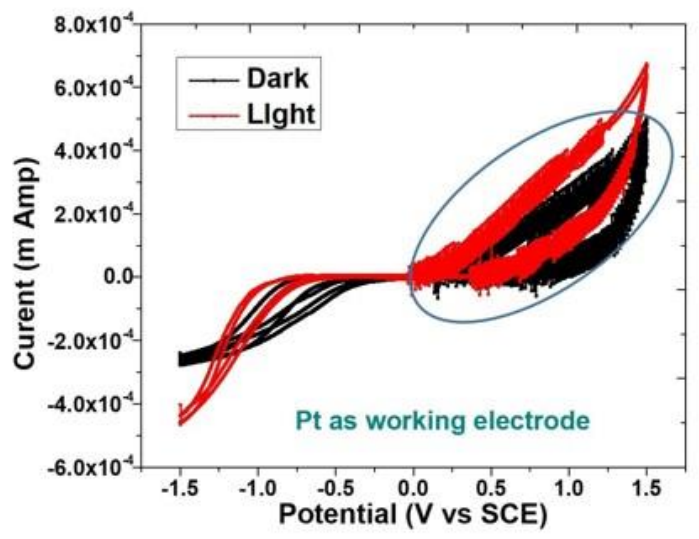

Figure 1. (A) Schematic of assembled liquid cell with integrated optical fiber for photo illumination. (B) Hummingbird Scientific liquid optical TEM holder. (C) MoS2 layers deposited on working electrode of the TEM liquid cell. (D) Comparative I-V characteristics of with and without illumination of light on the working electrode showing distinct redox peaks in the presence of light.

\section{References}

[1] F.M. Ross in Liquid Cell Electron Microscopy, Cambridge University Press (2016)

[2] Nowotny, J.; Sorrell, C. C.; Sheppard, L. R.; Bak, T. Int. J. Hydrogen Energy 2005, 30, 521

[3] Walter, M. G. et al. Solar water splitting cells. Chem. Rev. 110, 6446-6473 (2010)

[4] Maeda, K and Domen, K., J. Phys. Chem. Lett., 1(18), 2655-2661 (2010)

[5] Funding for the development of the sample holder was provided by the Department of Energy, Office of Basic Energy Sciences, through SBIR Grant \# DE-SC0015213 